\title{
In silico SNP analysis of the breast cancer antigen NY-BR-1
}

Zeynep Kosaloglu ${ }^{1,5 \dagger}$, Julia Bitzer ${ }^{2,5 \dagger}$, Niels Halama ${ }^{2,5}$, Zhiqin Huang ${ }^{3,6}$, Marc Zapatka ${ }^{3,6}$, Andreas Schneeweiss ${ }^{4,5}$, Dirk Jäger ${ }^{1,2,5}$ and Inka Zörnig ${ }^{2,5^{*}}$

\begin{abstract}
Background: Breast cancer is one of the most common malignancies with increasing incidences every year and a leading cause of death among women. Although early stage breast cancer can be effectively treated, there are limited numbers of treatment options available for patients with advanced and metastatic disease. The novel breast cancer associated antigen NY-BR-1 was identified by SEREX analysis and is expressed in the majority (>70\%) of breast tumors as well as metastases, in normal breast tissue, in testis and occasionally in prostate tissue. The biological function and regulation of NY-BR-1 is up to date unknown.

Methods: We performed an in silico analysis on the genetic variations of the NY-BR-1 gene using data available in public SNP databases and the tools SIFT, Polyphen and Provean to find possible functional SNPs. Additionally, we considered the allele frequency of the found damaging SNPs and also analyzed data from an in-house sequencing project of 55 breast cancer samples for recurring SNPs, recorded in dbSNP.
\end{abstract}

Results: Over 2800 SNPs are recorded in the dbSNP and NHLBI ESP databases for the NY-BR-1 gene. Of these, 65 (2.07\%) are synonymous SNPs, 191 (6.09\%) are non-synoymous SNPs, and 2430 (77.48\%) are noncoding intronic SNPs. As a result, 69 non-synoymous SNPs were predicted to be damaging by at least two, and 16 SNPs were predicted as damaging by all three of the used tools. The SNPs rs200639888, rs367841401 and rs377750885 were categorized as highly damaging by all three tools. Eight damaging SNPs are located in the ankyrin repeat domain (ANK), a domain known for its frequent involvement in protein-protein interactions. No distinctive features could be observed in the allele frequency of the analyzed SNPS.

Conclusion: Considering these results we expect to gain more insights into the variations of the NY-BR-1 gene and their possible impact on giving rise to splice variants and therefore influence the function of NY-BR-1 in healthy tissue as well as in breast cancer.

Keywords: NY-BR-1, Breast cancer, Antigen, SNPS, In silico

\section{Background}

Breast cancer is one of the most common malignancies and a leading cause of death among women. Although early stage breast cancer can be effectively treated, there are limited numbers of treatment options available for patients with advanced and metastatic disease. Therefore new targets and strategies need to be developed. A novel breast cancer differentiation antigen, designated as New

\footnotetext{
* Correspondence: Inka.Zoernig@nct-heidelberg.de

${ }^{\dagger}$ Equal contributors

2Department of Medical Oncology, National Center for Tumor Diseases (NCT) and University Hospital Heidelberg, Heidelberg, Germany

${ }^{5}$ Im Neuenheimer Feld 460, 69120 Heidelberg, Germany

Full list of author information is available at the end of the article
}

York-Breast-1 (NY-BR-1), was identified by a serological cloning strategy (SEREX) $[1,2]$ and could be a possible target for immunotherapy for breast cancer patients [3]. NY-BR-1, also known as ANKRD30A, is located on chromosome 10p11-p12. There are several transcripts existing, which contain between 36 and 42 exons. Although computational analyses have identified NY-BR-1 as being a potential transcription factor, the functional aspects of this $158.9 \mathrm{kDa}$ protein are still unknown. NYBR-1 protein was shown to be expressed in normal breast epithelia cells and in a majority of primary breast cancers [4, 5], while NY-BR-1 mRNA was detected predominantly in breast cancers [6, 7]. NY-BR-1 is over- 
expressed in over $70 \%$ of primary breast tumors and metastases [1] and additional details on the involvement of NY-BR-1 in breast cancer will lead to a better understanding of the underlying processes.

Genetic variation can have a major impact on gene function and the functional range of a gene cannot be fully understood without awareness of the potential variability within a gene [8]. To further understand the biological function and regulation of NY-BR-1 and its potential for therapeutic approaches, we performed an in silico analysis on the genetic variations of the NY-BR-1 gene.

Human genetic variants may occur in diverse nucleotide compositions, including single nucleotide polymorphisms (SNPs) and structural variants such as small insertions and deletions (indels) or large copy number variations. Among these, SNPs are the most prevalent form of human variation and it has been estimated that one SNP exists every 290 base-pairs in the human genome [9]. Evidences show that through SNPs a wide range of human diseases such as cancer or autoimmunity can be triggered $[10,11]$. SNPs also might affect the pharmacokinetics and pharmacodynamics of certain drugs in cancer therapy [12]. The transcriptional regulation of a protein, its structure and its function can be affected by a single base substitution, deletion or insertion. Two groups of SNPs are known: synonymous (sSNP) and non- synonymous SNPs (nsSNP). The latter results in changes of the translated amino acid sequence.

A number of studies have shown associations between one or few SNPs and complex diseases, but until today it is not entirely clear how much impact SNPs have on certain traits in different populations. With the steadily increasing number of known human nsSNPs, there is also growing interest in identification of the subset that may affect protein function. Various types of features can be used to predict the functional impact of nsSNPs: physical and chemical properties of the affected amino acids, structural properties of the encoded protein, and evolutionary properties, which can be inferred from sequence alignments of homologous proteins [13]. SIFT (Sorting Intolerant from Tolerant) [14], PROVEAN (Protein Variation Effect Analyzer) [15] and PolyPhen-2 (Polymorphism Phenotyping v2) [16] are computational prediction methods which take several of these properties into account and calculate a score to predict whether a given nsSNP has a functional impact. We obtained all SNPs for the NY-BR-1 gene and investigated the nsSNPs for their functional impact by using these three prediction tools. We identified a small number of nsSNPs which seem to affect the protein function of NY-BR-1. Additionally, we used in house sequencing data to analyze whether certain SNPs are enriched in breast cancer patients.

\section{Methods \\ SNP Mining}

dbSNP is hosted by the National Center for Biotechnology Information (NCBI) and is the largest repository of SNP data with over 140 million submitted variations [17].

Another source of variation data is provided by the "The National Heart, Lung and Blood Institute" (NHLBI). With the aim of discovering novel genes and mechanisms contributing to heart, lung and blood disorders, the NHLBI started the Exome Sequencing Project (ESP) and a large and well-phenotyped population with over 200,000 individuals was assembled. The protein coding regions of each individual genome (i.e. exome) is sequenced and the variation data is made publicly available [18].

The Ensembl Variation database incorporates variation data from several sources including dbSNP and NHLBI ESP. We used the web interface MartWizard (http://www.biomart.org/) of the BioMart Central Portal which offers access and crosslinks a wide array of biological databases.

The Ensembl transcript ID ENST00000611781 of the ANKRD30A gene was used to retrieve all available germline variations together with the corresponding genomic coordinates, the variant descriptions, the validation status, and allele frequency. Using the variant descriptions, we filtered coding non-synonymous SNPs (nsSNPs), coding synonymous SNPs (sSNPs) and intronic SNPs.

Additionally, exome-sequencing data were provided of 55 breast cancer patients from an in-house sequencing project (Division of Molecular Genetics, German Cancer Research Center (DKFZ), Heidelberg, Germany, and Heidelberg Center for Personalized Oncology (HIPO)). We also analyzed this dataset and looked for SNPs which are recorded in dbSNP.

\section{Prediction of the functional impact of coding nsSNPs using SIFT}

The prediction tool SIFT evaluates the functional impact of SNPs based on sequence homology. The prediction is based on the degree of conservation of each amino acid residue of the query sequence. To assess the degree of conservation, SIFT compiles a dataset of functionally related protein sequences by searching the protein databases UniProt and TrEMBL using the PSI-BLAST algorithm and builds an alignment of the found sequences and the query sequence. In the second step a normalized probability for each substitution at each position of the alignment is calculated and is then recorded in a scaled probability matrix. This scaled probability is also called the SIFT score and a substitution is considered to be tolerated if the score is greater than 0.05 ; those less than 0.05 are predicted to be deleterious. The SIFT approach assumes that a highly conserved position is intolerant to 
most substitutions, whereas a poorly conserved position can tolerate most substitutions.

\section{Prediction of the functional impact of coding nsSNPs using PROVEAN}

The tool PROVEAN also uses an alignment approach to assesses the functional impact of SNPs. PROVEAN consists of two main steps. In the first step, a set of homologous and distantly related sequences from the NCBI NR protein database is collected using BLASTP. To remove redundancy, the collected sequences are clustered, based on a sequence identity of $80 \%$. A so called supporting set of sequences is assembled by adding sequences from clusters most similar to the query sequence, until a sufficient number of clusters is reached in the supporting set. In the second step, for each sequence in the supporting sequence set, a delta score is computed using the BLOSUM62 substitution matrix. For each cluster, an average delta score is computed, and the averaged delta scores are again averaged among all clusters. This unbiased averaged delta score is the final PROVEAN score.

The PROVEAN approach assumes that a variation, which reduces similarity of protein A to the homologous or distantly related protein $\mathrm{B}$, is more likely to cause a damaging effect. Thus, the impact of a variation on protein function can be measured as the change in alignment score, the delta score. Low delta scores are interpreted as variations leading to a deleterious effect on protein function, while high delta scores are interpreted as variations with neutral effect.

The tools SIFT and PROVEAN are available online at http://sift.jcvi.org/ and http://provean.jcvi.org/, respectively. On the website, we used the tool PROVEAN Human Genome Variants, which provides PROVEAN and SIFT predictions for a list of human genome variants. We submitted the list of genomic coordinates and variants of our filtered 191 nsSNPs, and chose the default threshold of delta score $<=-2.5$ to detect deleterious variations.

\section{Prediction of the functional impact of coding nsSNPs using PolyPhen-2}

PolyPhen-2 combines information on sequence features, multiple alignments with homologous proteins, and structural parameters to predict the impact of a SNP on protein function.

For sequence-based assessment, PolyPhen-2 tries to identify the query as an entry in the UniProtKB/SwissProt database. Using the feature table of the corresponding entry, PolyPhen- 2 checks if a given SNP occurs at functional relevant site, e.g. if the SNP lies within a transmembrane, signal peptide, or binding region.

Similar to SIFT, PolyPhen-2 also assesses the degree of conversation of the position where the SNP occurs by utilizing a multiple sequence alignment of homologous sequences. For each variant PolyPhen-2 calculates a position-specific independent counts (PSIC) score. The PSIC score difference between the two variants describes the impact of a particular amino acid substitution: the higher the PSIC score difference, the higher functional impact the substitution is likely to have.

A BLAST query of the query sequence against protein structure databases is carried out to identify corresponding 3D protein structures. If corresponding structures are found, they are used to assess, whether the SNP is likely to destroy the hydrophobic core, interactions with ligands or other important features of the protein.

Finally, all parameters are taken together and empirical prediction rules are applied to make the final decision, whether the SNP is damaging or benign.

PolyPhen-2 is available online at http://genetics.bwh.harvard.edu/pph2/. We used the option 'Batch query' and submitted the list of genomic coordinates and variants of our filtered 191 nsSNPs.

\section{DNA Sequencing and Analysis}

The exon-sequencing library was prepared according to Agilent SureSelect Human All Exon V5 + UTRs protocol. Paired-end sequencing ( $2 * 101 \mathrm{bp})$ was carried out with Illumina Hiseq-2000 instruments. Paired-end sequencing reads were mapped to human genome reference assembly (hg19) with Burrows-Wheeler Aligner (BWA-v0.6.2) [19]. SAMtools mpileup (version-0.1.19) and bcftools (version-0.1.19) [20] were used to detect SNVs. Additional filtering step to remove possible artefacts was previously described [21]. Alignments on the NY-BR-1 gene only were extracted for this study and SNP states called at respective positions.

\section{Results \\ SNP Mining}

In the Ensembl BioMart database 2898 SNPs are recorded for the ANKRD30A transcript ENST00000611781. 2880 of these were imported from dbSNP and 18 from NHLBI ESP. 1832 SNPs have been validated by independent submissions or frequency/genotype data. However, the clinical significance has not been determined yet for any of the SNPs.

Out of all 2898 SNPs, 65 (2.07\%) were sSNPs, 191 (6.09\%) were nsSNPs, and 2430 (77.48\%) occurred in intronic regions (Fig. 1). 40 of the downloaded SNPs are annotated as splice region variants in dbSNP. We selected nsSNPs for our investigation.

\section{Deleterious nsSNPs predicted by SIFT}

Among the 191 analyzed nsSNPs, 79 nsSNPs were identified to be damaging with a tolerance index score $>=0.5$. Ten nsSNPS showed a highly damaging tolerance index score of 0.00 , namely rs200639888, 


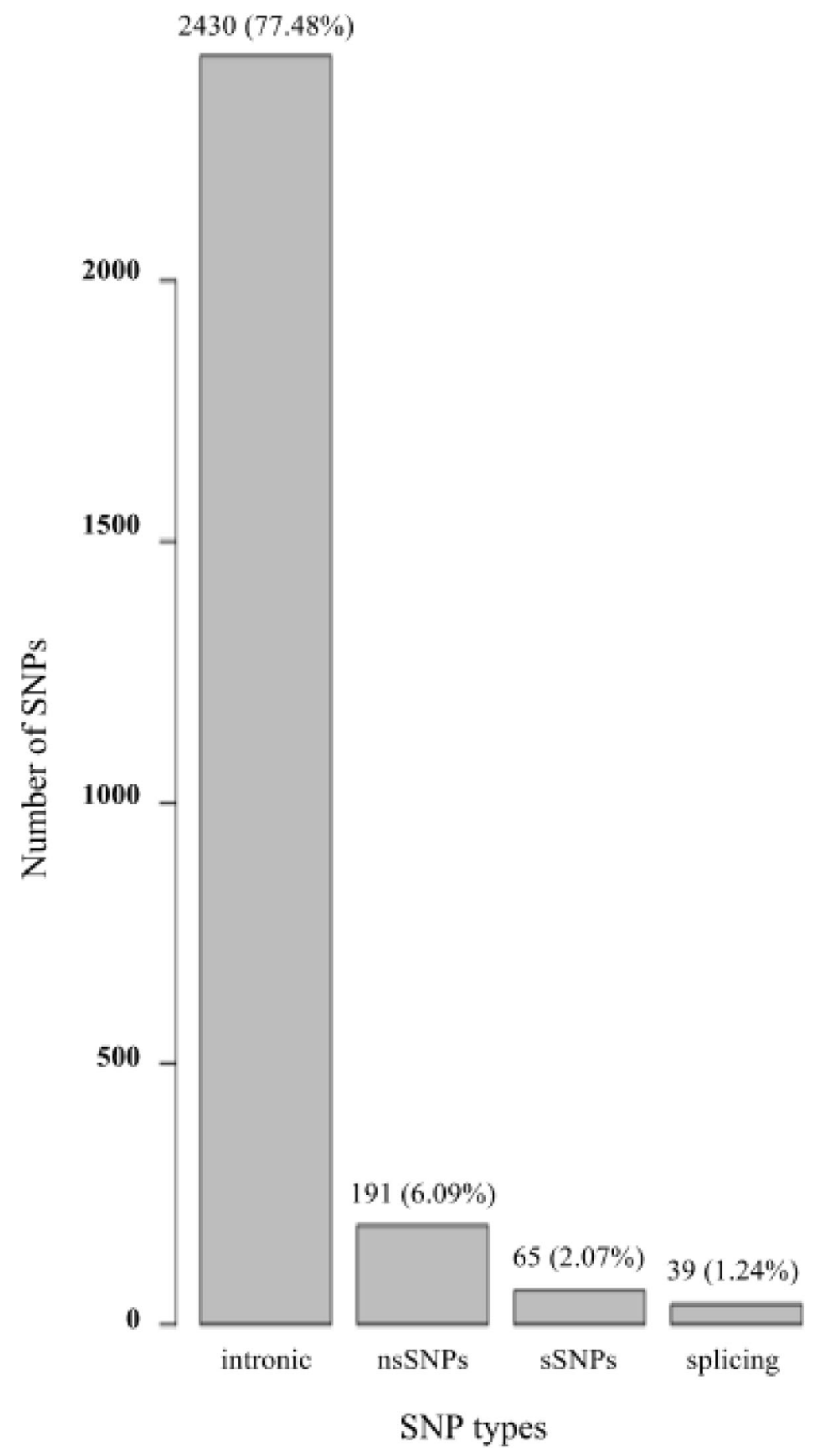

Fig. 1 Graphical representation of distribution of intronic SNPs, non-synoymous SNPs (nsSNPs), synonymous SNPs (sSNPs), and SNPs at splicing sites for the NY-BR-1 gene, based on the dbSNP and NHLBI ESP databases

rs372199195, rs144539033, rs369532435, rs199571878, rs376821949, rs267602482, rs201234943, rs367841401, and rs377750885. Nine nsSNPs had a tolerance index score of 0.001 , nine nsSNPs had a score of 0.002 , and five had a score of 0.003 . The remaining nsSNPs contained tolerance index scores varying between 0.004 and 0.048 .

\section{Damaging nsSNPs predicted by PROVEAN}

28 nsSNPs out of the analyzed 191 nsSNPs were predicted to be deleterious with a delta score of $<=-2.5$. 10 nsSNPs showed a highly deleterious score of $<-4.00$ : rs200639888 (-5.962), rs61737412(-5.030), rs201943652 (-4.758), rs189195791(-6.263), rs367841401 (-4.465), 
rs185294248(-4.366), rs374753521 (-4.184), rs371981 371 (-4.603), rs377750885(-5.87), and rs201764363 $(-4.025)$.

$20 \mathrm{nsSNPs}$ were predicted as damaging variations by SIFT and PROVEAN. rs200639888, rs367841401, and rs377750885 were predicted to be highly damaging by SIFT with a tolerance index score of 0.00 and are also predicted to be highly deleterious by PROVEAN with delta scores of -5.962 and -4.465 , and -5.87 respectively.

\section{Damaging nsSNPs predicted by PolyPhen}

Out of the 171 nsSNPs submitted to the PolyPhen-2 server, 102 nsSNPs were considered to be damaging: 44 nsSNPs were predicted to be 'probably damaging' with an PSIC score of 2.00 or more, and 58 nsSNPs were predicted to be 'possibly damaging' with an PSIC score of 1.40-1.90. The remaining 89 nsSNPs were predicted to be benign.

Sixty-four of the nsSNPs which were predicted to be damaging by SIFT, were also predicted damaging by PolyPhen. rs200639888, rs369532435, rs267602482, rs201234943, and rs377750885 were among the nsSNPs predicted to be highly damaging by SIFT with a tolerance index score of 0.00 . These five nsSNPs also have high PSIC scores predicted by PolyPhen $(2.439,2.746$, $2.23,2.373$, and 2.46 respectively).
19 nsSNPs were predicted to be damaging by Provean and PolyPhen, and 16 nsSNPs were predicted to be damaging by all three of the used tools (Fig. 2). The nsSNPs rs200639888, rs367841401, and rs377750885 were predicted to be highly damaging/deleterious by all three tools.

\section{Damaging nsSNPs predicted by at least two tools}

As summarized in Table 1, 16 nsSNPs were predicted damaging/deleterious by all three tools, and a total of 69 nsSNPs were predicted damaging by at least two of the used tools. We selected these 69 nsSNPs to perform a more detailed analysis.

Analysis of the spectrum of nsSNPs on the nucleotide level showed a conserved profile with $\mathrm{A}>\mathrm{T} / \mathrm{T}>\mathrm{A}$ transitions and hydrophile $>$ hydrophile transitions being the most frequent changes (Fig. 3a and b).

The nsSNPs rs200639888 and rs367841401, which were predicted to be highly damaging by all three tools, have an amino acid change from leucine to proline which are both hydrophobe amino acids. The third damaging nsSNP predicted by all three tools, rs377750885, has a change from glutamic acid (hydrophile) to (hydrophobe) valine.

The minor allele frequency describes the proportion of the least common allele in a certain population pool. Table 1 summarizes the minor allele frequency for the

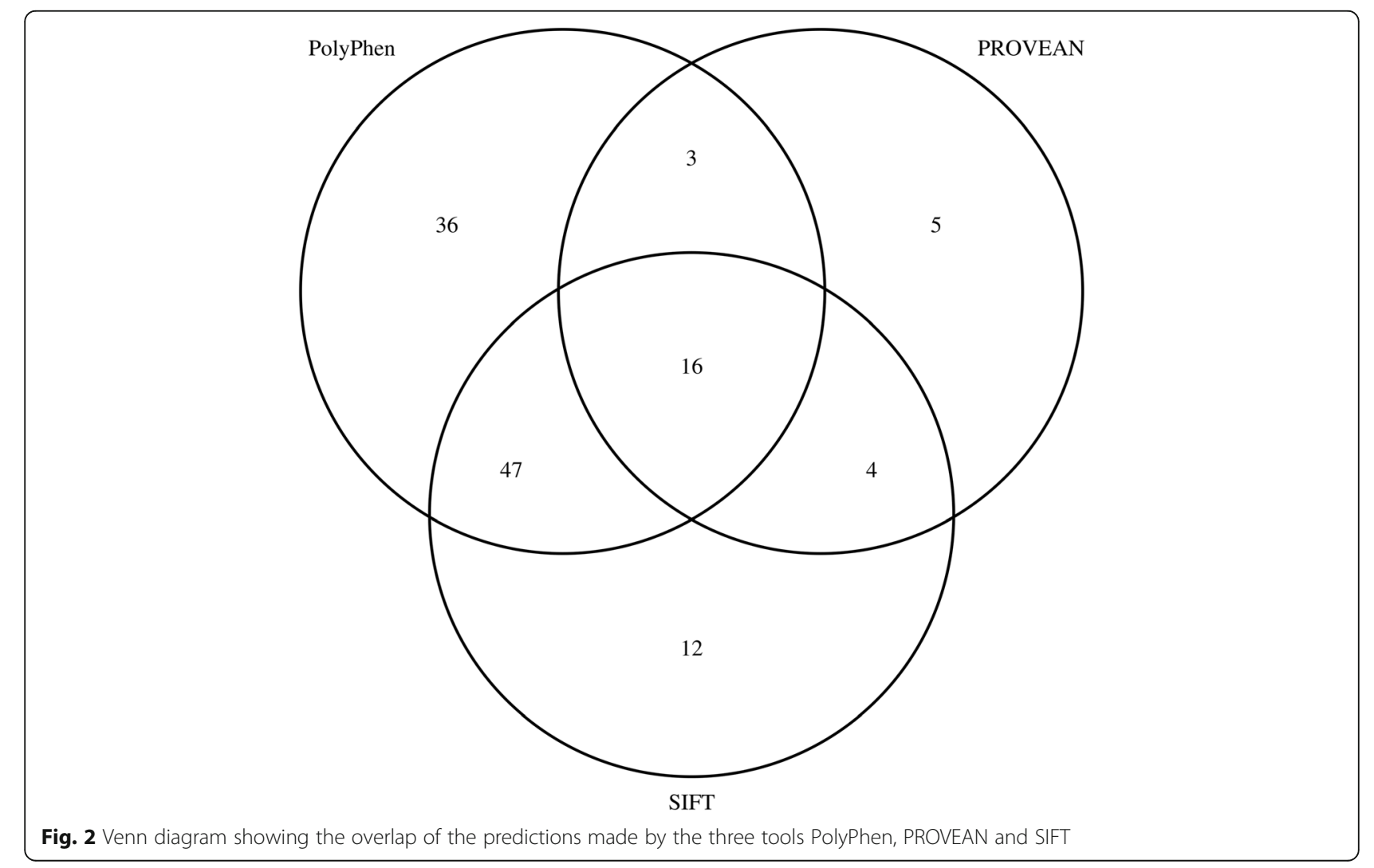


Table 1 Summary of all 69 nsSNPs predicted 504 to be damaging/deleterious by at least two of the used tools

\begin{tabular}{|c|c|c|c|c|c|c|c|c|c|c|}
\hline SNP ID & $\begin{array}{l}\text { Location on } \\
\text { chromosome }\end{array}$ & $\begin{array}{l}\text { Location in } \\
\text { protein }\end{array}$ & $\begin{array}{l}\text { Nucleotide } \\
\text { variation }\end{array}$ & $\begin{array}{l}\text { Protein } \\
\text { variation }\end{array}$ & SIFT prediction & $\begin{array}{l}\text { Provean } \\
\text { prediction }\end{array}$ & $\begin{array}{l}\text { Polyphen } \\
\text { prediction }\end{array}$ & AA change & $\begin{array}{l}\text { In ANK } \\
\text { domain }\end{array}$ & $\begin{array}{l}\text { Minor Allele } \\
\text { Frequency }\end{array}$ \\
\hline rs113525905 & 37447451 & 613 & $\mathrm{G} / \mathrm{A}$ & $G / R$ & Damaging(0.013) & Neutral(-1.25) & probably damaging $(0.998)$ & hydrophobe > hydrophile & & 0,19 \\
\hline rs1200875 & 37505192 & 985 & $C / T$ & $R / C$ & Damaging $(0.001)$ & Neutral(0.32) & possibly damaging $(0.762)$ & hydrophile > hydrophile & & 12,89 \\
\hline rs140013037 & 37505242 & 1001 & $\mathrm{G} / \mathrm{C}$ & $\mathrm{K} / \mathrm{N}$ & Damaging(0.016) & Deleterious(-3.58) & probably damaging $(0.963)$ & hydrophile > hydrophile & & 0,17 \\
\hline rs144539033 & 37430978 & 385 & $\mathrm{~T} / \mathrm{C}$ & $W / R$ & Damaging(0) & Neutral $(-1.04)$ & possibly damaging $(0.943)$ & hydrophobe > hydrophile & & 0,15 \\
\hline rs17590850 & 37470375 & 730 & $\mathrm{~A} / \mathrm{C}$ & $\mathrm{N} / \mathrm{H}$ & Damaging(0.002) & Neutral $(-0.35)$ & possibly damaging $(0.94)$ & hydrophile > hydrophile & & NA \\
\hline rs17606645 & 37470263 & 723 & $A / T$ & $\mathrm{~K} / \mathrm{N}$ & Damaging $(0.004)$ & Neutral(-0.44) & possibly damaging $(0.851)$ & hydrophile > hydrophile & & NA \\
\hline rs183760470 & 37451752 & 660 & $\mathrm{~A} / \mathrm{C}$ & K/Q & Damaging(0.002) & Neutral $(-0.78)$ & possibly damaging $(0.851)$ & hydrophile > hydrophile & & 0,01 \\
\hline rs184702413 & 37481992 & 838 & $\mathrm{~A} / \mathrm{G}$ & $E / G$ & Damaging(0.017) & Neutral(-1.16) & possibly damaging(0.851) & hydrophile > hydrophobe & & 0,41 \\
\hline rs185294248 & 37508038 & 1133 & $T / C$ & $\mathrm{~F} / \mathrm{S}$ & Damaging(0.012) & Deleterious(-4.37) & benign(0.006) & hydrophobe > hydrophile & & 0,11 \\
\hline rs190686350 & 37419160 & 122 & $\mathrm{G} / \mathrm{A}$ & $\mathrm{A} / \mathrm{T}$ & Damaging(0.012) & Deleterious(-3.27) & probably damaging $(0.997)$ & hydrophobe > hydrophile & Yes & 0,01 \\
\hline rs199571878 & 37438753 & 541 & $\mathrm{~A} / \mathrm{C}$ & $\mathrm{K} / \mathrm{Q}$ & Damaging(0) & Neutral $(-0.47)$ & possibly damaging(0.947) & hydrophile > hydrophile & & 0,079 \\
\hline rs199691521 & 37488715 & 926 & $A / T$ & EN & Damaging(0.02) & Neutral(-1.16) & probably damaging $(0.994)$ & hydrophile > hydrophobe & & NA \\
\hline rs199795040 & 37508139 & 1167 & $C / A$ & $\mathrm{Q} / \mathrm{K}$ & Damaging(0.002) & Neutral(-2.3) & possibly damaging $(0.886)$ & hydrophile > hydrophile & & NA \\
\hline rs199841724 & 37508538 & 1300 & $C / A$ & $\mathrm{H} / \mathrm{N}$ & Damaging(0.032) & Deleterious(-3.11) & benign(0.03) & hydrophile > hydrophile & & NA \\
\hline rs199874591 & 37451705 & 644 & $\mathrm{C} / \mathrm{A}$ & $\mathrm{P} / \mathrm{H}$ & Damaging(0.001) & Neutral(-1.11) & probably damaging $(0.997)$ & hydrophobe > hydrophile & & NA \\
\hline rs200114350 & 37486388 & 899 & $A / G$ & $N / S$ & Damaging(0.006) & Neutral(-1.64) & possibly damaging $(0.713)$ & hydrophile > hydrophile & & NA \\
\hline rs200264724 & 37431045 & 407 & $C / T$ & $\mathrm{~T} / \mathrm{M}$ & Damaging(0.001) & Neutral(0.55) & probably damaging $(0.989)$ & hydrophile > hydrophobe & & NA \\
\hline rs200331751 & 37478422 & 817 & $\mathrm{G} / \mathrm{A}$ & $\mathrm{D} / \mathrm{N}$ & Damaging(0.028) & Neutral $(-0.12)$ & possibly damaging $(0.818)$ & hydrophile > hydrophile & & NA \\
\hline rs200399695 & 37506718 & 1060 & $\mathrm{G} / \mathrm{C}$ & $R / T$ & Damaging(0.029) & Deleterious(-2.91) & benign(0.013) & hydrophile > hydrophile & & NA \\
\hline rs200639888 & 37419170 & 125 & $\mathrm{~T} / \mathrm{C}$ & $L / P$ & Damaging(0) & Deleterious(-5.96) & probably damaging $(0.997)$ & hydrophobe > hydrophobe & Yes & NA \\
\hline rs200651327 & 37418912 & 105 & $\mathrm{G} / \mathrm{A}$ & $E / K$ & Tolerated(0.081) & Deleterious(-3.37) & probably damaging $(0.999)$ & hydrophile > hydrophile & Yes & NA \\
\hline rs200845385 & 37430796 & 324 & $C / T$ & $\mathrm{~T} / \mathrm{I}$ & Damaging(0.002) & Neutral $(-0.78)$ & possibly damaging(0.898) & hydrophile > hydrophobe & & NA \\
\hline rs200929491 & 37508788 & 1383 & $\mathrm{G} / \mathrm{A}$ & $\mathrm{R} / \mathrm{H}$ & Damaging $(0.002)$ & Deleterious(-3.55) & probably damaging $(0.987)$ & hydrophile > hydrophile & & NA \\
\hline rs201234943 & 37447491 & 626 & $A / T$ & $\mathrm{~K} / \mathrm{M}$ & Damaging(0) & Neutral $(-1.29)$ & probably damaging(0.98) & hydrophile > hydrophobe & & 0,01 \\
\hline rs201628233 & 37478440 & 823 & $\mathrm{G} / \mathrm{T}$ & A/S & Damaging $(0.022)$ & Neutral $(-0.44)$ & possibly damaging(0.841) & hydrophobe $>$ hydrophile & & 0,39 \\
\hline rs201669885 & 37447325 & 602 & $C / G$ & P/A & Damaging(0.012) & Neutral $(-1.66)$ & possibly damaging $(0.924)$ & hydrophobe > hydrophobe & & 0,01 \\
\hline rs201764363 & 37508814 & 1392 & $\mathrm{G} / \mathrm{C}$ & A/P & Damaging $(0.002)$ & Deleterious(-4.03) & probably damaging $(0.969)$ & hydrophobe > hydrophobe & & 0,01 \\
\hline rs201858051 & 37508539 & 1300 & $\mathrm{~A} / \mathrm{G}$ & $H / R$ & Tolerated(0.108) & Deleterious(-3.06) & possibly damaging(0.651) & hydrophile > hydrophile & & NA \\
\hline rs201885728 & 37451744 & 657 & $\mathrm{~T} / \mathrm{C}$ & $L / S$ & Damaging(0.01) & Neutral $(-0.08)$ & possibly damaging $(0.932)$ & hydrophobe > hydrophile & & 0,01 \\
\hline rs201943652 & 37421175 & 173 & $\mathrm{~T} / \mathrm{C}$ & $L / P$ & Damaging(0.011) & Deleterious(-4.76) & probably damaging $(0.995)$ & hydrophobe > hydrophobe & Yes & NA \\
\hline rs201976592 & 37447446 & 611 & $\mathrm{C} / \mathrm{A}$ & $\mathrm{T} / \mathrm{N}$ & Damaging(0.002) & Neutral $(-1.14)$ & possibly damaging $(0.851)$ & hydrophile > hydrophile & & 0,05 \\
\hline rs202090351 & 37430699 & 292 & $C / A$ & $P / T$ & Damaging $(0.001)$ & Neutral(-1.91) & probably damaging $(0.998)$ & hydrophobe > hydrophile & & NA \\
\hline rs202098264 & 37430875 & 350 & $\mathrm{C} / \mathrm{G}$ & F/L & Damaging $(0.003)$ & Neutral(-1.01) & probably damaging $(0.965)$ & hydrophobe > hydrophobe & & NA \\
\hline rs202200263 & 37454055 & 679 & $\mathrm{~A} / \mathrm{G}$ & $D / G$ & Damaging $(0.001)$ & Neutral(-1.04) & possibly damaging $(0.924)$ & hydrophile > hydrophobe & & 0,01 \\
\hline
\end{tabular}


Table 1 Summary of all 69 nsSNPs predicted 504 to be damaging/deleterious by at least two of the used tools (Continued)

\begin{tabular}{|c|c|c|c|c|c|c|c|c|c|c|}
\hline rs267602477 & 37419220 & 142 & $\mathrm{G} / \mathrm{A}$ & $A / T$ & Damaging $(0.016)$ & Deleterious(-3.41) & probably damaging $(0.997)$ & hydrophobe > hydrophile & Yes & NA \\
\hline rs267602481 & 37438727 & 532 & $C / T$ & $S / F$ & Damaging(0.001) & Neutral $(-1.27)$ & possibly damaging(0.842) & hydrophile > hydrophobe & & NA \\
\hline rs 267602482 & 37441009 & 556 & $C / T$ & $S / F$ & Damaging(0) & Neutral(-1.66) & probably damaging(0.99) & hydrophile > hydrophobe & & NA \\
\hline rs267602485 & 37507968 & 1110 & G/A & $E / K$ & Damaging(0.021) & Deleterious(-3.34) & probably damaging(0.98) & hydrophile > hydrophile & & NA \\
\hline rs367841401 & 37508002 & 1121 & $\mathrm{~T} / \mathrm{C}$ & $L / P$ & Damaging(0) & Deleterious(-4.47) & probably damaging $(0.969)$ & hydrophobe > hydrophobe & & NA \\
\hline rs368559588 & 37508121 & 1161 & G/A & $\mathrm{A} / \mathrm{T}$ & Damaging(0.04) & Neutral $(-0.75)$ & possibly damaging(0.618) & hydrophobe > hydrophile & & 0,05 \\
\hline rs368660392 & 37442552 & 587 & $\mathrm{~A} / \mathrm{G}$ & $H / R$ & Damaging $(0.003)$ & Neutral(-1.4) & possibly damaging $(0.932)$ & hydrophile > hydrophile & & 0,01 \\
\hline rs369099906 & 37508651 & 1337 & $\mathrm{~A} / \mathrm{T}$ & $L / F$ & Damaging(0.001) & Deleterious(-3.22) & probably damaging $(0.999)$ & hydrophobe > hydrophobe & & NA \\
\hline rs369118323 & 37422851 & 209 & $C / T$ & $L / F$ & Damaging(0.01) & Neutral(-1.52) & probably damaging $(0.993)$ & hydrophobe > hydrophobe & Yes & NA \\
\hline rs369532435 & 37438591 & 519 & $\mathrm{~A} / \mathrm{T}$ & K/M & Damaging(0) & Neutral(-1.13) & probably damaging $(0.996)$ & hydrophile > hydrophobe & & 0,01 \\
\hline rs371253665 & 37451583 & 636 & $C / T$ & P/L & Damaging(0.001) & Neutral $(-0.77)$ & probably damaging $(0.994)$ & hydrophobe > hydrophobe & & 0,01 \\
\hline rs371384886 & 37430859 & 345 & $C / T$ & AN & Damaging $(0.001)$ & Neutral $(-0.56)$ & probably damaging $(0.997)$ & hydrophobe > hydrophobe & & 0,01 \\
\hline rs371443557 & 37431010 & 395 & $\mathrm{~T} / \mathrm{G}$ & $1 / M$ & Damaging(0.004) & Neutral $(-0.28)$ & possibly damaging(0.676) & hydrophobe > hydrophobe & & NA \\
\hline rs371878855 & 37508548 & 1303 & $A / G$ & $\mathrm{Q} / \mathrm{R}$ & Damaging(0.012) & Deleterious(-2.52) & possibly damaging $(0.808)$ & hydrophile > hydrophile & & NA \\
\hline rs371981371 & 37508671 & 1344 & $C / A$ & $A / D$ & Damaging(0.003) & Deleterious(-4.6) & probably damaging $(0.989)$ & hydrophobe > hydrophile & & NA \\
\hline rs372199195 & 37430803 & 326 & $\mathrm{~T} / \mathrm{G}$ & $D / E$ & Damaging(0) & Neutral(-0.13) & possibly damaging $(0.643)$ & hydrophile > hydrophile & & NA \\
\hline rs372420008 & 37430922 & 366 & $\mathrm{~A} / \mathrm{G}$ & $K / R$ & Damaging $(0.007)$ & Neutral(-0.62) & possibly damaging $(0.956)$ & hydrophile > hydrophile & & NA \\
\hline rs372878721 & 37442530 & 580 & $G / A$ & V/M & Damaging $(0.013)$ & Neutral $(-0.77)$ & probably damaging $(0.976)$ & hydrophobe > hydrophobe & & NA \\
\hline rs373377344 & 37508379 & 1247 & $G / A$ & $E / K$ & Damaging $(0.048)$ & Deleterious(-2.78) & possibly damaging $(0.898)$ & hydrophile > hydrophile & & NA \\
\hline rs373380909 & 37422972 & 249 & $\mathrm{G} / \mathrm{T}$ & $G / N$ & Damaging $(0.003)$ & Neutral(-2.41) & probably damaging $(0.999)$ & hydrophobe > hydrophobe & Yes & NA \\
\hline rs373997768 & 37505217 & 993 & $\mathrm{~A} / \mathrm{C}$ & $\mathrm{K} / \mathrm{T}$ & Damaging $(0.003)$ & Deleterious(-2.76) & probably damaging $(0.963)$ & hydrophile > hydrophile & & NA \\
\hline rs374024060 & 37430943 & 373 & $\mathrm{C} / \mathrm{T}$ & $\mathrm{T} / \mathrm{M}$ & Damaging $(0.011)$ & Neutral $(-0.76)$ & probably damaging $(0.975)$ & hydrophile > hydrophobe & & NA \\
\hline rs374037740 & 37441038 & 566 & $T / G$ & W/G & Damaging(0.009) & Neutral(-1.82) & possibly damaging $(0.826)$ & hydrophobe > hydrophobe & & NA \\
\hline rs374739457 & 37454063 & 682 & $\mathrm{G} / \mathrm{C}$ & $E / Q$ & Damaging(0.018) & Neutral $(-0.76)$ & possibly damaging $(0.851)$ & hydrophile > hydrophile & & NA \\
\hline rs374753521 & 37508446 & 1269 & $\mathrm{~A} / \mathrm{C}$ & Y/S & Damaging $(0.031)$ & Deleterious(-4.18) & benign $(0.347)$ & hydrophile > hydrophile & & NA \\
\hline rs375945698 & 37505306 & 1023 & $\mathrm{G} / \mathrm{C}$ & $E / Q$ & Damaging(0.018) & Neutral(-2.17) & probably damaging $(0.999)$ & hydrophile > hydrophile & & NA \\
\hline rs376116213 & 37505157 & 973 & $\mathrm{G} / \mathrm{A}$ & $R / K$ & Damaging $(0.004)$ & Neutral $(-2.23)$ & probably damaging $(0.976)$ & hydrophile > hydrophile & & 0,01 \\
\hline rs376821949 & 37438772 & 547 & $\mathrm{G} / \mathrm{A}$ & $R / K$ & Damaging(0) & Neutral $(0.04)$ & possibly damaging $(0.643)$ & hydrophile > hydrophile & & NA \\
\hline rs377410013 & 37440994 & 551 & $\mathrm{~T} / \mathrm{C}$ & $M / T$ & Damaging $(0.045)$ & Neutral $(-0.42)$ & possibly damaging $(0.717)$ & hydrophobe > hydrophile & & NA \\
\hline rs377740138 & 37430720 & 299 & $\mathrm{G} / \mathrm{A}$ & V/M & Damaging $(0.002)$ & Neutral(-0.39) & possibly damaging $(0.845)$ & hydrophobe > hydrophobe & & NA \\
\hline rs377744149 & 37508352 & 1238 & $\mathrm{G} / \mathrm{A}$ & $\mathrm{D} / \mathrm{N}$ & Tolerated(0.083) & Deleterious(-3.19) & probably damaging $(0.971)$ & hydrophile > hydrophile & & 0,01 \\
\hline rs377750885 & 37508803 & 1388 & $\mathrm{~A} / \mathrm{T}$ & EN & Damaging(0) & Deleterious(-5.87) & probably damaging $(0.997)$ & hydrophile > hydrophobe & & NA \\
\hline rs41276130 & 37451768 & 665 & $\mathrm{~T} / \mathrm{G}$ & L/W & Damaging(0.002) & Neutral(-0.98) & probably damaging $(0.983)$ & hydrophobe > hydrophobe & & 4,17 \\
\hline rs45515098 & 37440991 & 550 & $C / T$ & $\mathrm{P} / \mathrm{L}$ & Damaging(0.028) & Neutral $(-1.27)$ & possibly damaging(0.581) & hydrophobe > hydrophobe & & 0,01 \\
\hline rs61737412 & 37419218 & 141 & $\mathrm{C} / \mathrm{T}$ & T/M & Damaging $(0.035)$ & Deleterious(-5.03) & possibly damaging $(0.951)$ & hydrophile > hydrophobe & Yes & 4,13 \\
\hline
\end{tabular}



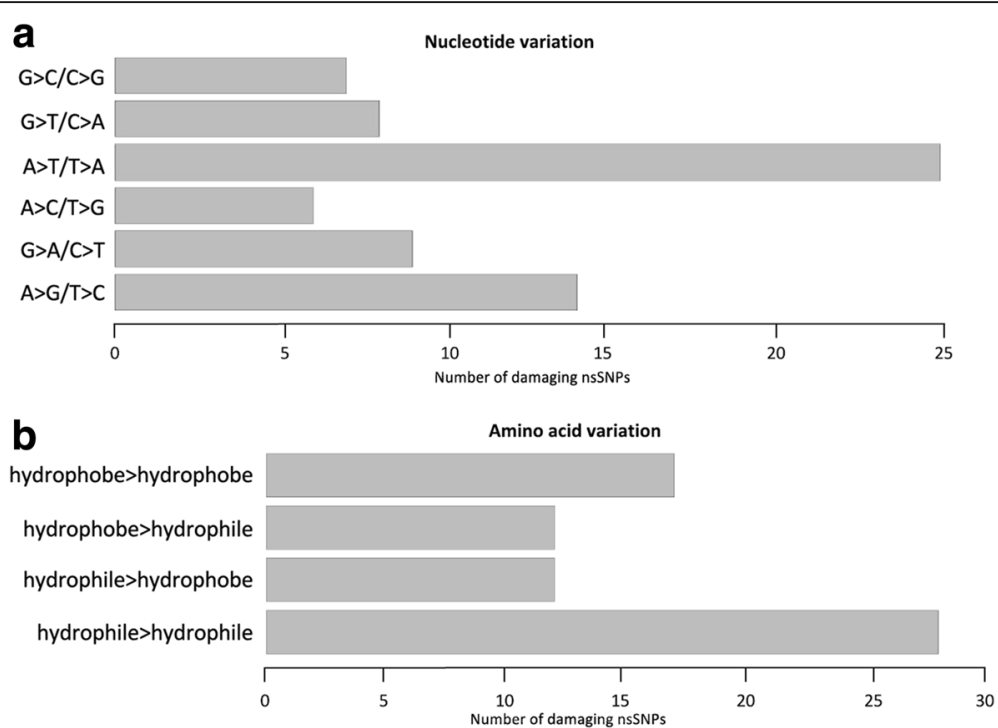

Fig. 3 Graphical representation of spectrum of damaging nsSNPs variation. a) nucleotide variations, b) amino acid variations

69 nsSNPs, predicted to be deleterious by two tools. Allele frequencies are only provided for 26 of the analyzed SNPs. For most SNPs the minor allele frequency is below 1\% except for the SNPs rs1200875 (12.9\%), rs41276130 (4.17\%), and rs61737412 (4.13\%).

\section{Clinical data analysis}

As part of an in-house cancer sequencing project, exome-sequencing data was available for 55 breast cancer patients and was provided for analysis in this study. In the analyzed patient cohort 11 SNPs were detected in in the NY-BR-1 gene: rs34042320, rs1209750, rs34552 277, rs61737412, rs41276130, rs1200876, rs1200875, rs4 1304589, rs116939015, and rs16937417 (Table 2). Seven
SNPs occur in more than 10 patients and three of these (rs61737412, rs41276130, rs1200875) were predicted damaging by at least two of the used tools. These SNPs also have a high minor allele frequency of $4.13,4.17$, and 12.89 , respectively. The SNP rs1209750 occurs in 49 patients, which corresponds to almost $90 \%$ of analyzed patients. rs1209750 also has a high minor allele frequency of $48,22 \%$. This SNP however, was not predicted to be damaging. Likewise, the SNPs rs1200876, rs34042320, and rs34552277 occur in a large fraction of the patient cohort and also have a high minor allele frequency. These SNPs were also not predicted to be damaging. A Fisher's exact test was performed to test the difference in allele frequencies of the SNPs in our breast

Table 2 SNPs and mutations detected in the analyzed breast cancer patient cohort of 55 patients

\begin{tabular}{|c|c|c|c|c|}
\hline SNP ID & Prediction & Frequency in Patient Cohort & Minor Allele Frequency in dbSNP & $\mathrm{p}$-value (Fisher's exact test) \\
\hline rs116939015 & & 1,82 & 0,59 & 0,4921 \\
\hline rs1200875 & damaging & 45,45 & 12,89 & 0,0018 \\
\hline rs1200876 & & 45,45 & 12,77 & 0,0016 \\
\hline rs1209750 & & 89,09 & 48,22 & 0,0149 \\
\hline rs12766884 & & 1,82 & 4,432 & 0,0853 \\
\hline rs16937417 & & 1,82 & 2,61 & 0,3646 \\
\hline rs34042320 & & 18,18 & 3,27 & 0,0033 \\
\hline rs34552277 & & 38,18 & 22,76 & 0,3381 \\
\hline rs41276130 & damaging & 21,82 & 4,17 & 0,0020 \\
\hline rs41304589 & & 9,09 & 2,55 & 0,2059 \\
\hline rs61737412 & damaging & 27,27 & 4,13 & $3,80 \mathrm{E}-05$ \\
\hline somatic (chr10:37430943:C/T) & & 1,82 & NA & \\
\hline somatic (chr10:37447328:A/G) & & 1,82 & NA & \\
\hline
\end{tabular}


cancer patient cohort against the dbSNP reference for significance which showed six SNPs to be significantly enriched in the analyzed patient cohort.

Two somatic mutations were also detected in the patient cohort which both occur only in single patients. The somatic mutation chr10:37430943: $\mathrm{C}>\mathrm{T}$ translates to a $\mathrm{T}>\mathrm{M}$ transition at position 317 in the NY-BR-1 protein. This mutation is also documented in the Catalogue of Somatic Mutations in Cancer (COSMIC) [22] database as COSM4137978 and was reported in two patients with ovary cancer. The second somatic mutation chr10:37447328: A > G translates to a N > D transition at protein position 547 and is not documented in the COSMIC database.

\section{Discussion}

Information on genetic variation can provide a valuable insight into the functional range and critical regions of a gene. SNPs are the most common form of genetic variations and a link between SNPs and complex diseases have been reported for a number of cases. The BRCA-1 gene for example and some of its interaction partners are associated with breast cancer. SNPs in these genes are not just involved in the onset of a disease but they can promote also disease progression and outcome $[23,24]$. Here, we systematically analyzed SNPs in the NY-BR-1 gene to identify those SNPs which can modify the functional properties of the protein.

In the Ensembl BioMart database 2898 SNPs are recorded for the NY-BR-1 transcript ENST00000611781. Out of these, 191 (6.01\%) were nonsynonymous SNPs (nsSNPs), i.e. polymorphisms which translate into an altered amino acid sequence. As these types of SNPs are most likely to have an effect on protein function, we chose to analyze only them further.

Computational approaches use various types of features to predict the functional impact of nsSNPs: physical and chemical properties of the affected amino acids, structural properties of the encoded protein, and evolutionary properties, which can be inferred from sequence alignments of homologous proteins. We chose three state-of-the-art computational tools which can predict the effects of amino acid substitutions on protein function: SIFT, Provean and PolyPhen2.

191 nsSNPs were analyzed and the results varied between the used tools: SIFT predicted 79 damaging nsSNPs, Provean 28 nsSNPs, and PolyPhen2 102 nsSNPs. 16 nsSNPs were predicted damaging by all three tools, and a total of 69 nsSNPs were predicted damaging by at least two of the used tools. SIFT and PolyPhen2 have the biggest overlap with 63 common predictions. This may be due to the common step of assessing the degree of conversation by utilizing a multiple sequence alignment of homologous sequences. 36 damaging
nsSNPs were only predicted by PolyPhen 2 because PolyPhen2 is the only tool that takes functional relevant sites into account. The location of the 69 damaging SNPS within the ANKRD30A gene is shown in Fig. 4a.

Up to date the structure of NY-BR-1 has not been solved yet and no homology models for the entire protein are available. Thus, we unfortunately could not evaluate the location and effect of the predicted damaging nsSNPs on the protein structure.

In the UniProt database six ankyrin (ANK) repeat motifs are documented for NY-BR-1. The ANK repeat motif is one of the most common protein-protein interaction motifs in nature and occurs in a large number of functionally diverse proteins. The structure of the ANK repeat motif is conserved: each repeat typically consists of 30-34 amino acid residues comprising two anti-parallel $\alpha$-helices and a long loop ending in a $\beta$-hairpin [25]. Proteins containing the ANK repeat motif are involved in a diverse set of cellular functions, and defects in ANK repeat proteins have been associated with a number of human diseases [26, 27]. Hence, a variation within such a functional domain is likely to have an impact on protein function.

Eight of the 69 damaging nsSNPs in NY-BR-1 are located in an ANK repeat motif: rs190686350, rs20063 9888, rs61737412, rs267602477, rs201943652, rs2006 51327, rs369118323, rs373380909. PolyPhen2 predicted all of them as damaging, whereas Provean predicted two, and SIFT one of them as not damaging. SNPs influencing the splicing process also may have an impact on protein function if the newly generated transcripts are translated into proteins. In dbSNP, 39 of the NY-BR-1 SNPs are annotated to be splicing, located at donor or acceptor sites (Fig. 4b). These SNPs have the potential to influence the splicing process and thus give rise to new transcripts.

An unknown fraction of SNPs submitted to the public databases may not be true polymorphisms, but examples of sequencing errors. Therefore it is important to consider the validation status of each SNP. A polymorphism can be validated by independent submissions or frequency/genotype data. In our dataset 1832 out of 2898 SNPs have been validated. Considering the 69 damaging nsSNPs, 16 have not been validated yet. As these nsSNPs seem to have an impact on protein function, validation of them should especially be considered.

Allele frequencies are only provided for 26 of the analyzed SNPs 69 nsSNPs, predicted to be damaging by at least two tools. SNPs with no information on allele frequencies are usually based on single submissions, often from sequencing projects of cancer patient cohorts and therefore might be of special relevance. The minor allele frequency of 16 out of 26 analyzed SNPs is below $0.1 \%$, for six analyzed SNPs the minor allele frequencies are 


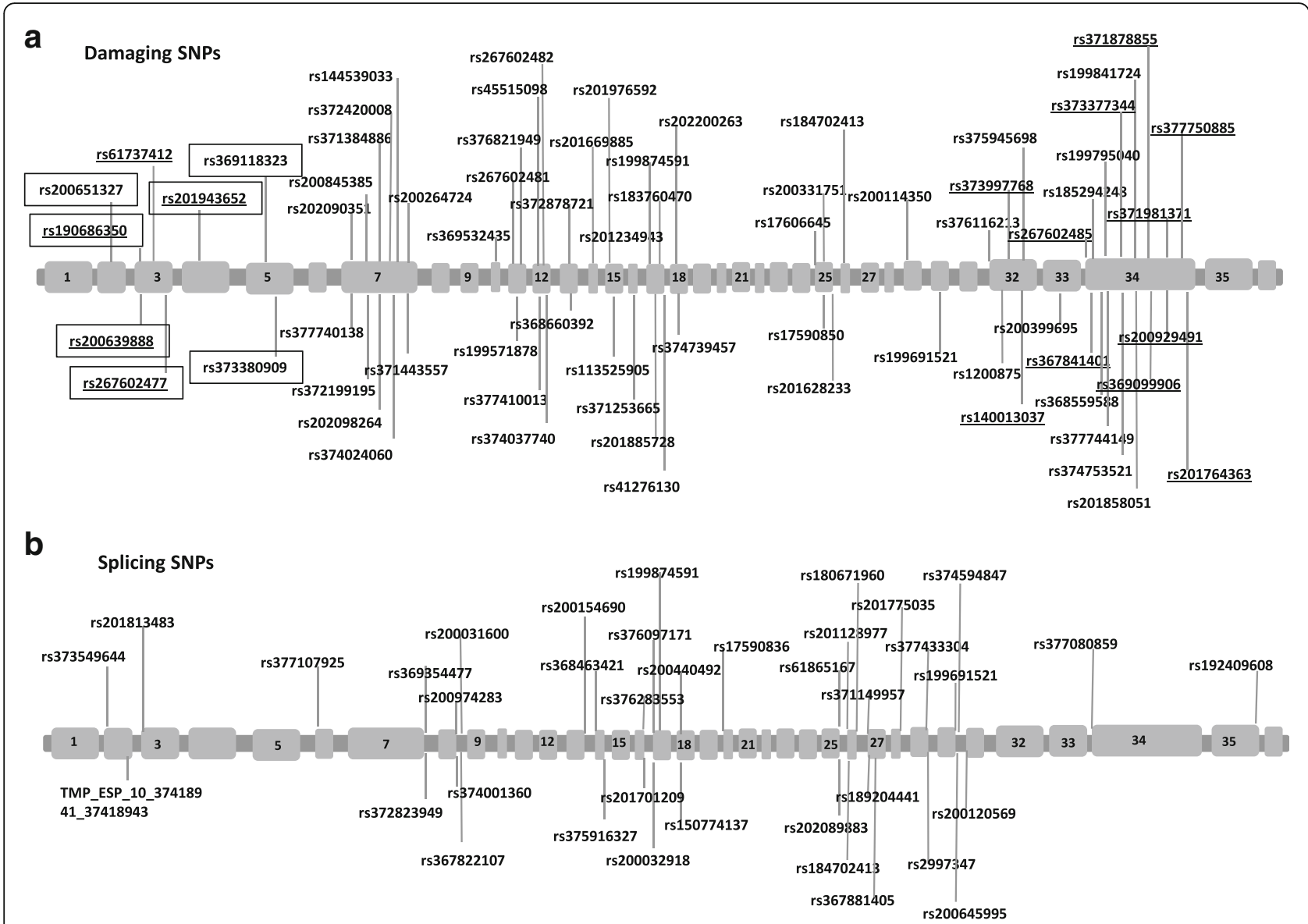

Fig. 4 Graphical representation of location of NY-BR-1 SNPS. a) damaging SNPS, b) splicing SNP. SNPs predicted damaging by all three tools are underlined and SNPs located in an ANK repeat domain are highlighted with a box

between $0.1 \%$ and $1 \%$, and for three SNPs the minor allele frequency is greater than $4 \%$ According to Frazer et al. these SNPs can be classified according to their minor allele frequencies: variants with minor allele frequencies between $0.1 \%$ and $3 \%$ were defined as rare variants, variants with minor allele frequencies of less than $0.1 \%$ as novel variants, and high-frequency common variants were defined as variants with minor allele frequencies greater than $5 \%$ [28].

We also analyzed in house exome-sequencing data of 55 breast cancer patients and as expected, NYBR1 was found to be expressed in all patients (data not shown). Somatic mutations were only detected in two patients. Also, as indicated by the database research on COSMIC, the two somatic mutations are not being frequently observed in cancer patients. Only one of the mutations, COSM4137978, is documented in COSMIC and was observed in two cases of ovary cancer. Also using the COSMIC database, we further searched for NY-BR-1 mutations in breast cancer patients. Only 27 out of 1436 breast cancer patients were found to have a somatic mutation in NY-BR-1 indicating that somatic mutations in this gene is not a frequent event in breast cancer patients.

In contrast, 11 SNPs in the NY-BR-1 gene were found in the in-house patient cohort, seven SNPs occurring in more than 10 patients. Three of these frequent SNPs (rs61737412, rs41276130, rs1200875) were also predicted damaging by at least two of the used tools. These SNPs also have a high minor allele frequency in dbSNP, they are however highly enriched in the patient cohort ( $\mathrm{p}$ value $<0.002$, Fisher's exact test). There are also three other SNPs (rs1200876, rs1209750, rs34042320), that are enriched in the patient cohort ( $\mathrm{p}$-value $<0.01$, Fisher's exact test), but these SNPs were not predicted damaging. These SNPs which seem to be enriched in breast cancer patients need to be further analyzed in larger patient cohorts to elucidate whether there is a correlation to clinical status and outcome. The effect of these SNPs on protein function also still needs to be determined.

\section{Conclusion}

In summary, we have identified 69 damaging nsSNPs within the coding region of the breast cancer associated 
NY-BR-1 gene. Moreover, we found 39 potential splicing SNPs which can affect the alternative splicing process. Our analysis gives an overview on the SNP landscape of NYBR1 and now provides the basis to further study the association of SNPs and the molecular breast cancer subtypes "Her2", "Luminal A/B" and "Triple negative" as well as clinical data, such as treatment response, relapse rate and overall survival.

\section{Abbreviations}

ANK: Ankyrin; indels: Small insertions and deletions; NY-BR-1: New YorkBreast-1; SEREX: Serological cloning strategy; SNP: Single Nucleotide Polymorphism

\section{Acknowledgment}

We thank the DKFZ-Heidelberg Center for Personalized Oncology (DKFZ-HIPO) for technical support and funding through HIP0017 project number (AS, MZ, ZH). JB was partially funded by the NCT Interdisciplinary Research Program.

\section{Availability of data and material}

The RNASeq data from the HIPO project will be publicly accessible as soon as the main study is published.

\section{Authors' contributions}

$\mathrm{NH}, \mathrm{DJ}$ and IZ conceived of and designed this study. IZ coordinated the study and helped to draft the manuscript. $\mathrm{ZK}, \mathrm{ZH}$ and $\mathrm{NH}$, carried out the bioinformatic analysis. JB helped to analyse and interpret the data. JB and ZK drafted the manuscript. AS provided samples (HIPO project). MZ acquired the data. AS, DJ and $M Z$ critically revised the manuscript. All authors read and approved the final manuscript.

\section{Competing interests}

The authors declare that they have no competing interests.

\section{Consent for publication}

By giving their informed consent (Ethic's vote S-039/2008) the patients also gave their consent for the publication of the data.

\section{Ethics approval and consent to participate}

The study was approved by the Medical Ethics Committee of the University of Heidelberg (S-039/2008). Tumor samples were used after receiving a signed informed consent from the patients.

\section{Author details \\ ${ }^{1}$ Clinical Cooperation Unit "Applied Tumor Immunity", National Center for Tumor Diseases (NCT) and German Cancer Research Center (DKFZ), Heidelberg, Germany. ²Department of Medical Oncology, National Center for Tumor Diseases (NCT) and University Hospital Heidelberg, Heidelberg, Germany. ${ }^{3}$ Division of Molecular Genetics, German Cancer Research Center (DKFZ), Heidelberg, Germany. ${ }^{4}$ Department of Obstetrics and Gynecology, National Center for Tumor Diseases (NCT) and University Hospital Heidelberg, Heidelberg, Germany. ${ }^{5}$ Im Neuenheimer Feld 460, 69120 Heidelberg, Germany. ${ }^{6}$ Im Neuenheimer Feld 580, 69120 Heidelberg, Germany.}

Received: 3 August 2016 Accepted: 3 November 2016 Published online: 18 November 2016

\section{References}

1. Jager D, Karbach J, Pauligk C, Seil I, Frei C, Chen YT, et al. Humoral and cellular immune responses against the breast cancer antigen NY-BR-1: definition of two HLA-A2 restricted peptide epitopes. Cancer Immun. 2005;5:11.

2. Jager D, Stockert E, Gure AO, Scanlan MJ, Karbach J, Jager E, et al. Identification of a tissue-specific putative transcription factor in breast tissue by serological screening of a breast cancer library. Cancer Res. 2001;61(5): 2055-61.

3. Seil I, Frei C, Sultmann H, Knauer SK, Engels K, Jager E, et al. The differentiation antigen NY-BR-1 is a potential target for antibody-based therapies in breast cancer. Int J Cancer. 2007;120(12):2635-42. doi:10.1002/ ijc.22620.
4. Jager D, Filonenko V, Gout I, Frosina D, Eastlake-Wade S, Castelli S, et al. NY-BR-1 is a differentiation antigen of the mammary gland. Appl Immunohistochem Mol Morphol. 2007;15(1):77-83.

5. Varga Z, Theurillat JP, Filonenko V, Sasse B, Odermatt B, Jungbluth AA, et al. Preferential nuclear and cytoplasmic NY-BR-1 protein expression in primary breast cancer and lymph node metastases. Clin Cancer Res. 2006;12(9): 2745-51. doi:10.1158/1078-0432.CCR-05-2192.

6. Jiang Y, Harlocker SL, Molesh DA, Dillon DC, Stolk JA, Houghton RL, et al. Discovery of differentially expressed genes in human breast cancer using subtracted cDNA libraries and CDNA microarrays. Oncogene. 2002;21(14): 2270-82. doi:10.1038/sj.onc.1205278.

7. Nissan A, Jager D, Roystacher M, Prus D, Peretz T, Eisenberg I, et al. Multimarker RT-PCR assay for the detection of minimal residual disease in sentinel lymph nodes of breast cancer patients. Br J Cancer. 2006;94(5): 681-5. doi:10.1038/sj.bjc.6602992.

8. Barnes MR. Genetic variation analysis for biomedical researchers: a primer. Methods Mol Biol. 2010;628:1-20. doi:10.1007/978-1-60327-367-1_1.

9. Kruglyak L, Nickerson DA. Variation is the spice of life. Nat Genet. 2001;27(3): 234-6. doi:10.1038/85776.

10. Xavier RJ, Rioux JD. Genome-wide association studies: a new window into immune-mediated diseases. Nat Rev Immunol. 2008:8(8):631-43. doi:10. 1038/nri2361.

11. Bodmer W, Bonilla C. Common and rare variants in multifactorial susceptibility to common diseases. Nat Genet. 2008;40(6):695-701. doi:10. 1038/ng.f.136

12. Wang JB, Pang GSY, Chong SS, Lee CGL. SNP web resources and their potential applications in personalized medicine. Curr Drug Metab. 2012; 13(7):978-90.

13. Nakken S, Alseth I, Rognes T. Computational prediction of the effects of non-synonymous single nucleotide polymorphisms in human DNA repair genes. Neuroscience. 2007;145(4):1273-9. doi:10.1016/j.neuroscience.2006. 09.004 .

14. Kumar P, Henikoff S, Ng PC. Predicting the effects of coding non-synonymous variants on protein function using the SIFT algorithm. Nat Protoc. 2009;4(7): 1073-82. doi:10.1038/nprot.2009.86.

15. Choi $Y$, Sims GE, Murphy S, Miller JR, Chan AP. Predicting the functional effect of amino acid substitutions and indels. Plos One. 2012;7(10):e46688. doi:10.1371/journal.pone.0046688.

16. Adzhubei IA, Schmidt S, Peshkin L, Ramensky VE, Gerasimova A, Bork P, et al. A method and server for predicting damaging missense mutations. Nat Methods. 2010;7(4):248-9. doi:10.1038/nmeth0410-248.

17. Sherry ST, Ward MH, Kholodov M, Baker J, Phan L, Smigielski EM, et al. dbSNP: the NCBI database of genetic variation. Nucleic Acids Res. 2001; 29(1):308-11. doi:10.1093/Nar/29.1.308.

18. Dorschner MO, Amendola LM, Turner EH, Robertson PD, Shirts BH, Gallego CJ, et al. Actionable, pathogenic incidental findings in 1,000 participants exomes. Am J Hum Genet. 2013;93(4):631-40. doi:10.1016/j.ajhg.2013.08.006.

19. Li H, Durbin R. Fast and accurate long-read alignment with Burrows-Wheeler transform. Bioinformatics. 2010;26(5):589-95. doi:10.1093/bioinformatics/btp698.

20. Li H, Handsaker B, Wysoker A, Fennell T, Ruan J, Homer N, et al. The sequence alignment/map format and SAMtools. Bioinformatics. 2009;25(16):2078-9. doi: 10.1093/bioinformatics/btp352.

21. Jones DT, Jager N, Kool M, Zichner T, Hutter B, Sultan M, et al. Dissecting the genomic complexity underlying medulloblastoma. Nature. 2012; 488(7409):100-5. doi:10.1038/nature11284.

22. Forbes SA, Beare D, Gunasekaran P, Leung K, Bindal N, Boutselakis $H$, et al. COSMIC: exploring the world's knowledge of somatic mutations in human cancer. Nucleic Acids Res. 2015;43(Database issue):D805-11. doi: 10.1093/nar/gku1075

23. Alshatwi AA, Hasan TN, Syed NA, Shafi G, Grace BL. Identification of functional SNPs in BARD1 gene and in silico analysis of damaging snps: based on data procured from dbSNP database. Plos One. 2012;7(10) e43939. doi:10.1371/journal.pone.0043939.

24. Johnson N, Fletcher O, Palles C, Rudd M, Webb E, Sellick G, et al. Counting potentially functional variants in BRCA1, BRCA2 and ATM predicts breast cancer susceptibility. Hum Mol Genet. 2007;16(9):1051-7. doi:10.1093/Hmg/ Ddm050.

25. Chakrabarty B, Parekh N. Identifying tandem Ankyrin repeats in protein structures. BMC bioinformatics. 2014;15(1):6599. doi:10.1186/s12859-014-0440-9.

26. Leite RC, Basseres DS, Ferreira JS, Alberto FL, Costa FF, Saad ST. Low frequency of ankyrin mutations in hereditary spherocytosis: identification of 
three novel mutations. Hum Mutat. 2000;16(6):529. doi:10.1002/10981004(200012)16:6<529::AID-HUMU13>3.0.CO;2-N.

27. Li J, Mahajan A, Tsai MD. Ankyrin repeat: a unique motif mediating protein-protein interactions. Biochemistry. 2006;45(51):15168-78. doi:10. 1021/bi062188q.

28. Frazer KA, Murray SS, Schork NJ, Topol EJ. Human genetic variation and its contribution to complex traits. Nat Rev Genet. 2009;10(4):241-51. doi:10. 1038/nrg2554

Submit your next manuscript to BioMed Central and we will help you at every step:

- We accept pre-submission inquiries

- Our selector tool helps you to find the most relevant journal

- We provide round the clock customer support

- Convenient online submission

- Thorough peer review

- Inclusion in PubMed and all major indexing services

- Maximum visibility for your research

Submit your manuscript at www.biomedcentral.com/submit 